\title{
Cerebellar-Dependent Learning in Larval Zebrafish
}

\author{
Mark Aizenberg ${ }^{1,2}$ and Erin M. Schuman ${ }^{1,2}$ \\ ${ }^{1}$ California Institute of Technology, Division of Biology, Pasadena, California 91125, and ${ }^{2}$ Max Planck Institute for Brain Research, 60438 Frankfurt, \\ Germany
}

Understanding how neuronal network activity contributes to memory formation is challenged by the complexity of most brain circuits and the restricted ability to monitor the activity of neuronal populations in vivo. The developing zebrafish (Danio rerio) is an animal model that circumvents these problems, because zebrafish larvae possess a rich behavioral repertoire and an accessible brain. Here, we developed a classical conditioning paradigm in which 6- to 8-d-old larvae develop an enhanced motor response to a visual stimulus (conditioned stimulus, CS) when it is paired with touch (unconditioned stimulus, US). Using in vivo calcium imaging we demonstrate that CS and US activate different subsets of neurons in the cerebellum; their activity, modulated by learning two-photon laser ablation, revealed that the cerebellum is involved in acquisition and extinction, but not the retention, of this memory.

\section{Introduction}

Many of the same features that make zebrafish an attractive system for studies of neuronal development also facilitate the study of the neural bases of behavior. Namely, zebrafish can learn (Darland and Dowling, 2001; Sison and Gerlai, 2010) and are amenable to neurodevelopmental and neurogenetic analysis and manipulation (Asakawa et al., 2008; Baier and Scott, 2009). Even in an early stage of development zebrafish larvae possess a wide spectrum of innate and experience-dependent behaviors (Burgess and Granato, 2007; Gerlach et al., 2008). Importantly, their relatively small size, manageable number of neurons, and optical accessibility make them attractive for studying the neural mechanisms of behavior (Friedrich et al., 2010). Despite these clear advantages, there exist very few examples of learning paradigms in larval fish.

In this article, we developed a classical conditioning paradigm in which zebrafish larvae learned to associate a light stimulus (conditioned stimulus, CS) with a tactile stimulus (unconditioned stimulus, US), resulting in robust associative conditioning. During conditioning, we imaged spontaneous and evoked neural activity in the cerebellum. Our results demonstrate that multimodal stimuli converge to form high-order representations in the cerebellum. We identified a subset of neurons in the corpus cerebelli (CCe) of the cerebellum that reflect associative learning as an increased response to the CS. Selective lesions indicate that the CCe is required for both the acquisition and extinction of conditioned memory.

Received Dec. 16, 2010; revised March 25, 2011; accepted April 15, 2011.

Author contributions:M.A. and E.M.S. designed research;M.A. performed research;M.A. analyzed data;M.A. and E.M.S. wrote the paper.

Funding from the Gordon and Betty Moore Foundation supported this work. We gratefully acknowledge Ueli Rutishauser, Flora Hinz, and Gilles Laurent for comments on the manuscript. We thank Sally Kim for help with the imaging set-up and all Schuman group members for helpful discussions and criticism.

Correspondence should be addressed to Erin M. Schuman, Max Planck Institute for Brain Research, 60438 Frankfurt, Germany. E-mail: schumane@brain.mpg.de.

DOI:10.1523/JNEUROSCI.6565-10.2011

Copyright $\odot 2011$ the authors $\quad 0270-6474 / 11 / 318708-05 \$ 15.00 / 0$

\section{Materials and Methods}

Animals and imaging. All of our experiments, unless specified otherwise, were performed on the nacre strain of zebrafish (6-8 d postfertilization), kindly provided by D. Prober (California Institute of Technology). Larvae were raised at $28^{\circ} \mathrm{C}$ on a $14 \mathrm{~h}: 10 \mathrm{~h}$ light/dark cycle in E3 solution. For bolus injections, larvae were anesthetized using $0.01 \%$ tricaine and embedded in 1.5\% low-melting agarose. Oregon Green BAPTA-1 AM Ester (OGB1-AM; Invitrogen) was dissolved at a concentration of $1 \mathrm{~mm}$ in DMSO with $20 \%$ pluronic acid (Invitrogen) and diluted to a concentration of $40 \mu \mathrm{M}$ in E3. Using a pulled glass pipette, larvae were bilaterally injected with OGB1-AM into the CCe. Fish were then released and allowed to recover for at least $1 \mathrm{~h}$. For imaging, individual fish were embedded in 1.5\% low-melting agarose (SeaPlaque Agarose, Cambrex) in a small dish filled with fresh E3 $(\sim 5 \mathrm{ml})$. Agarose covering the tail was carefully removed to allow the tail to move freely. Imaging of intracellular $\mathrm{Ca}^{2+}$ fluctuations was accomplished using $20 \times 1.0 \mathrm{NA}$ water-immersion objective lens and a LSM 510 Meta confocal laser scanning microscope (Carl Zeiss MicroImaging). Scans were performed with a $4 \mathrm{~Hz}$ rate at a depth of $40 \pm 5 \mu \mathrm{m}$ from the surface of the brain unless otherwise specified. A custom-made objective inverter was used in combination with an inverted microscope to make possible video recordings.

Two-photon laser ablation. To identify the CCe for ablation, fish injected with OGB1-AM were imaged under the conditions described above. Laser ablation was accomplished using a similar setup but with a tunable mode-locked Ti:Sapphire laser pumped by a $15-\mathrm{W}$ pump laser (Spectra Physics). The mode-locked laser produced $80-150 \mathrm{fs}$ pulses at a repetition rate of $80 \mathrm{MHz}$ with a center wavelength of $800 \mathrm{~nm}$. The average power at the specimen plane used for these experiments was 3000 $\mathrm{mW}$. Ablations were performed using 50 bidirectional scans of the targeted region. To verify tissue ablation fish were stained in whole-mount protocol with SYTO 14 green fluorescent nucleic acid stain (Invitrogen). Briefly, ablated fish were fixed in $4 \%$ PFA, permeabilized with Proteinase $\mathrm{K}(10 \mu \mathrm{g} / \mathrm{ml}$; Roche $)$ for $1 \mathrm{~h}$, thoroughly washed with PBS-Tween 20 , and incubated overnight with SYTO 14 green diluted 1:10,000.

Imaging data analysis. Confocal stacks were analyzed offline by custom-written software (Matlab, Mathworks). To outline the somata of active neurons, an automatic customized segmentation algorithm based on the fluctuation in the pixel fluorescence $\left(\Delta F / F_{\min }\right)$ was applied to the image series, where $F_{\min }$ is the minimal value in the given signal trace. Segmented elements were then refined manually to separate merged cells or discard neurons that did not exhibit $\mathrm{Ca}^{2+}$ transient higher than 
A
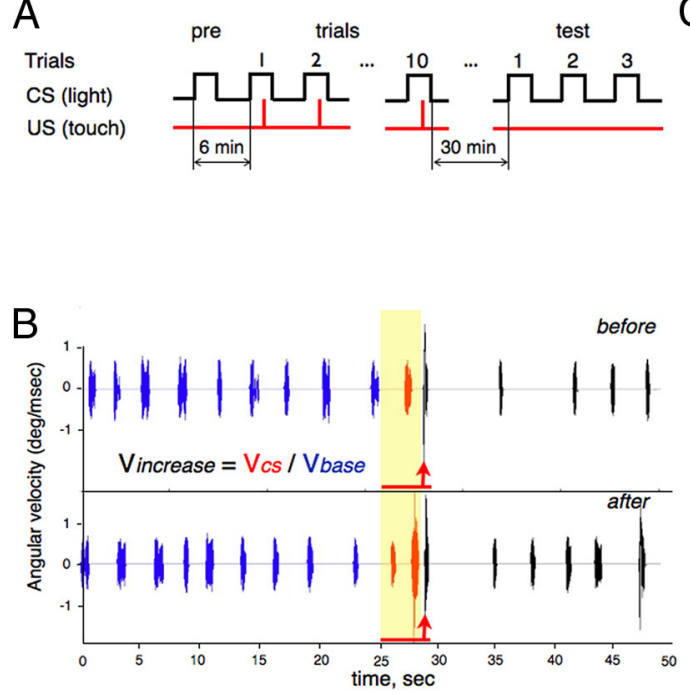

Figure 1. Classical conditioning in larval zebrafish. $A$, Conditioning protocol. In the first trial (pre) a single CS was presented to examine the animal's response to the $C S$ alone. Ten paired $C S+U S$ trials were presented with a 6 min intertrial interval (ITI). Three test trials (CS alone) were presented after various delays. B, Sample analysis of conditioned and unconditioned responses (CR and $U R$, respectively). The tail angular velocity before (upper trace) and after (lower trace) learning was compared. The UR can be seen after US presentation (red arrows). The red line represents the CS. C, The effect of paired (CS+US), unpaired (CS + US unpaired), and $C S$ alone trials on the CR. Each point represents the mean $V_{\text {increase }}$ value for all fish in a given trial $\pm S E M$. Conditioning resulted in a significantly enhanced $C R$ that grew in magnitude over the 10 trials and was rapidly extinguished by three test trials. Training with CS alone or unpaired CS + US did not significantly alter the CR. D, Memory decline over time. Fish were conditioned, and each group was tested twice (ITI $=4 \mathrm{~min}$ ) either 5, 30, or $60 \mathrm{~min}$ after the last trial. Each bar represents mean $V_{\text {increase }}$ value for two trials \pm SEM. ${ }^{*} p<0.05$.

$F_{\text {mean }}+(1.5 \times \sigma)$, where $F_{\text {mean }}$ is the average fluorescence. Cells that displayed slow transients $\left(\tau_{\text {decay }}>5\right.$ s) typical of glial cells were also excluded from analysis. Trials that showed drift in the $z$-axis as result of movement artifacts were removed from further analysis. Shifts in the $x-y$ plane were compensated for semiautomatically using Matlab. To examine the activity of a single neuron during many trials, stacks from trials were concatenated and aligned to compensate for inconsistency in the $x-y$ plane.

For quantitative analysis of responses to the CS or US, the raw fluorescent signal was processed to reconstruct the firing rate as described previously (Yaksi and Friedrich, 2006). Briefly, data were first normalized to a baseline. Next, the fluorescent response was smoothed according to a custom algorithm and deconvolved with an exponential kernel $\left(\tau_{\text {decay }}=3 \mathrm{~s}\right)$. Finally, peaks of activity were detected using a customized Matlab function. A neuron was considered as responsive to light if for at least three trials the average peak height represented a $10 \%$ or more increase over the baseline; the peaks were aligned within a $0.5 \mathrm{~s}$ time window. Cross-correlation analyses were computed using Matlab's cross-covariance function. The correlation sequence was then normalized to obtain correlation coefficient values between -1 and 1 .

Behavioral setup and analysis, learning protocol. Fish were partially restrained in low melting point agarose to allow their tails to move freely and then placed in the center of a $60-\mathrm{mm}$-diameter circular array of 32 LEDs. Visual stimuli were provided unilaterally by sequential presentation of flashes of a white light in a rostrocaudal direction if not specified otherwise. The choice of a moving spot over a flash of light was motivated by the fact that the former is less likely to elicit unconditioned tail response, thus being more behaviorally "neutral." Each LED was on for $0.25 \mathrm{~s}$, which made the entire CS duration equal to $4 \mathrm{~s}$. The light flux density of the LED, as measured from a distance of $10 \mathrm{~mm}$, was $50 \mu \mathrm{W}$. Touch stimulation was provided by a stainless-steel wire positioned close to the side of the body, driven by a piezoelectric manipulator (PM-10, WPI). Tail locomotion was monitored using high-speed video recording (Redlake MotionXtra camera at $125 \mathrm{~Hz}$ frame rate) and analyzed offline using custom-written Matlab software. The stimulus parameters and high-speed camera operation were controlled by a computer using custom-written software (Labview, National Instruments).
Angular velocity $(V)$ at each frame was obtained by taking the first derivative of the tail's orientation. Activity of a subject during a relevant period of time was defined as the sum of absolute values of $V$ for corresponding frames. The $\mathrm{CR}$ was defined as an increase in the activity ( $\left.V_{\text {increase }}\right)$ during the CS but before the US presentation $\left(V_{\mathrm{CS}}\right)$ over the activity preceding the CS $\left(V_{\text {base }}\right): V_{\text {increase }}=V_{c s} / V_{\text {base }}$.

On occasion, we observed that laser light sometimes caused a temporary increase in fish motor behavior. We therefore did not use data collected in the first $15 \mathrm{~s}$ for the calculation of $V_{\text {base }}$. To examine the correlation between the tail velocity of a subject with the $\mathrm{Ca}^{2+}$ signal of the neurons, the velocity trace was transformed by taking its absolute value, resampling to a lower sample rate, and convolving the data with a filter kernel $\left(\tau_{\text {decay }}=3 \mathrm{~s}\right)$.

In the behavioral protocol, only fish that exhibited spontaneous locomotion in at least $50 \%$ of trials were used for statistical analysis. Fish were pretested for responses to the CS. Only fish that did not display a strong response to the CS $\left(V_{\text {increase }}<2\right)$ were used in experiments. In total, $\sim 40 \%$ of the fish were excluded from the statistics according to these criteria. Then, the conditioned group received 10 paired CS and US presented with a 6 min ITI. Three test trials (CS alone) were presented after $30 \mathrm{~min}$, unless specified otherwise. Two control groups received either the same number of CSs and USs in quasi-random manner or no US at all. In the experiments with ablations, after lesions, fish were left for $30 \mathrm{~min}$ in agarose to recover.

Statistical analysis. Nonparametric statistics were used for most behavioral analyses. For comparisons of samples from two or more groups, Mann-Whitney and Kruskal-Wallis (KW) tests were used. For pairwise comparisons, the Wilcoxon rank-sum test was used. For experiments where the sample size exceeded 20 or for evaluation of an interaction effect, parametric tests were applied (one- and two-way ANOVA, $t$ test). A $\chi^{2}$ test was used to compare proportions.

\section{Results}

\section{Classical conditioning in larval zebrafish}

We developed a Pavlovian conditioning paradigm in 6- to 8- $\mathrm{d}$ old zebrafish in which the head of each fish was embedded in agarose to enable the measurement of neuronal $\mathrm{Ca}^{2+}$ signals while a high-speed camera recorded the freely moving tail. In the learning protocol (Fig. 1A), a visual CS was provided by a circular array of LEDs that delivered a spot of white light that moved in a horizontal plane either rostral-to-caudal or vice versa. Fish were trained to associate the CS with a swift touch to a side of the body (US), resulting in an enhanced tail movement upon exposure to the light (conditioned response; CR) (Fig. $1 B, C$ ). We quantified the CR ( $V_{\text {increase; }}$ see Materials and Methods) as the fold increase in velocity during the CS $\left(V_{\mathrm{CS}}\right)$ over spontaneous swim $\left(V_{\text {base }}\right)$ $($ Fig. $1 B$ ). After repeated pairings of the CS and US, a significant CR was acquired within seven trials ( $n=21, p<0.001$, KW test). In contrast, neither exposure to CS alone nor unpaired presentations of the CS and US resulted in a significant increase in tail velocity ( $n=16$ and $12, p=0.28$ and 0.25 , respectively, KW test). To assess memory retention, we presented the CS alone in three postconditioning test sessions (T1-T3). Thirty minutes after the last pairing trial, a CR was still evident (test 1) but underwent extinction over the two subsequent presentations of the CS alone $(n=11, p=0.036, \mathrm{KW}$ test) (Fig. $1 C)$. To examine the duration 


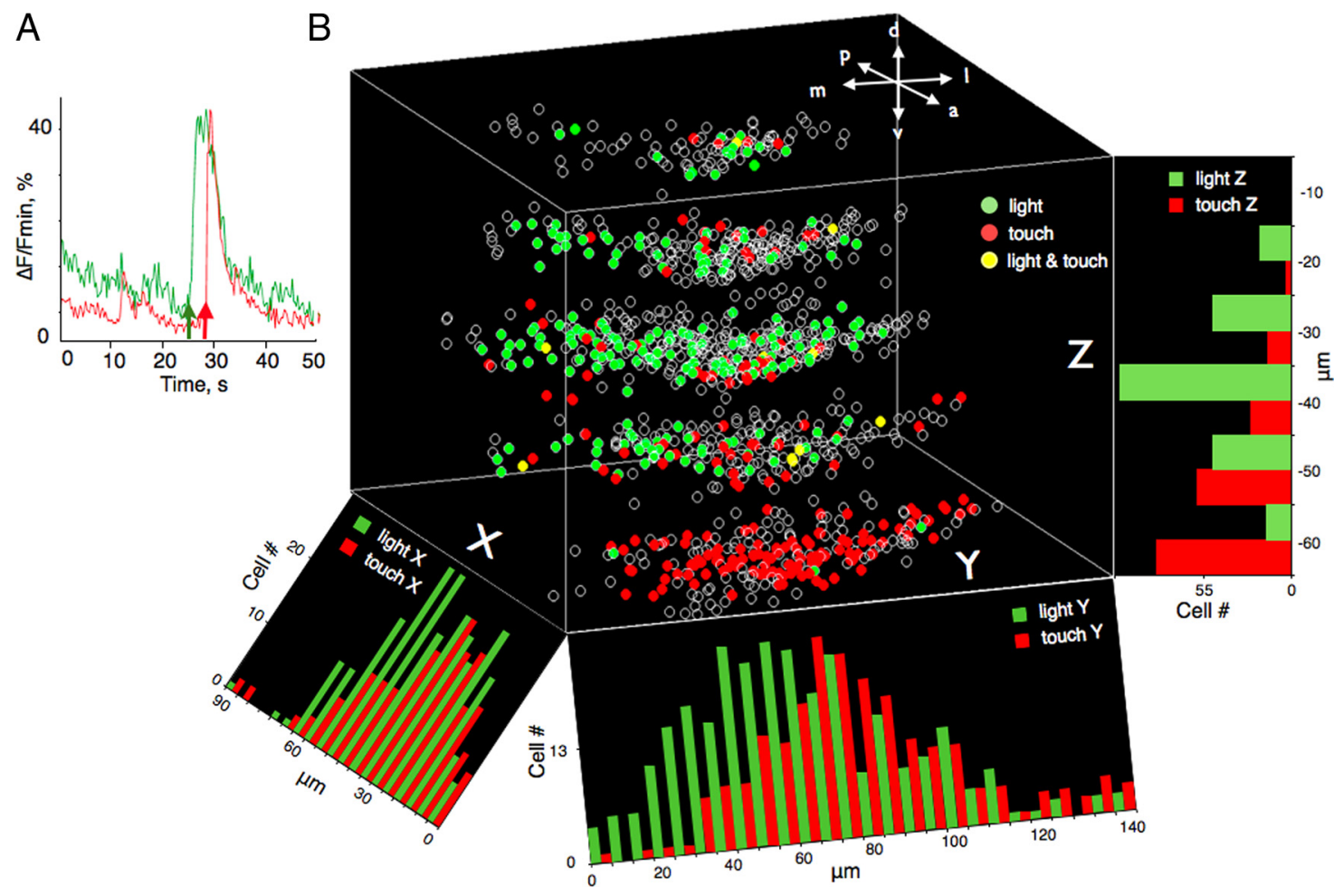

Figure 2. $\quad \boldsymbol{A}$, Change in fluorescence averaged across 25 light- and touch-evoked cerebellar neurons (green and red lines, respectively) as a function of time. The green arrow represents the onset of the visual stimulus (CS). The red arrow depicts the timing of touch delivery. $\boldsymbol{B}$, Three-dimensional functional map of the left cerebellar hemisphere. The zero point on the $z$-axis is located at the dorsal surface of the cerebellum. Each $z$-section is separated by $10 \mu \mathrm{m}$. The intersection between $x$ - and $y$-axes is the point where the midline between the hemispheres crosses the border to the optic tectum. Each circle depicts the position of an individual neuron. Green circles represent the light-driven neurons. Red circles represent touch-evoked neurons. Yellow circles represent neurons that responded to both visual and tactile stimuli. Orientation is as indicated: a, anterior; p, posterior; $m$, medial; $\mathrm{l}$, lateral; $\mathrm{d}$, dorsal, v, ventral. Histograms depict the density of light- and touch-evoked neurons (green and red bars, respectively) projected on $x-, y$-, and $z$-axes. Note that the density of touch-driven cells gradually increases in the dorsolateral direction, whereas light-evoked neurons are most densely packed at a depth of $40 \mu \mathrm{m}$.

of memory retention we halted the pairings and waited variable periods of time. Memory steadily declined over 5, 30, and $60 \mathrm{~min}$ after the last conditioning trial (Fig. $1 D)(n=10$ for each group, $p=0.011, \mathrm{KW}$ test). Thus, this learning in larval zebrafish is characterized by rapid acquisition and extinction and relatively short-term retention of memory.

\section{CS- and US-evoked activity in the cerebellum}

The cerebellum is the site of convergence of CS-, US-, and CRrelated circuitry essential for classical conditioning of many motor responses in different species (Thompson and Steinmetz, 2009). To better understand the functional organization of the larval zebrafish cerebellum, we measured spontaneous and evoked neural activity in vivo using confocal imaging in CCe bolus-loaded with a $\mathrm{Ca}^{2+}$ indicator (OGB1-AM) while simultaneously monitoring tail movements. We first compared the $\mathrm{Ca}^{2+}$ activity of individual neurons and the simultaneously acquired tail locomotion and assigned each neuron with a correlation coefficient value (see Materials and Methods). In the absence of explicit stimulation, the cerebellum showed relatively sparse spontaneous activity and a weak correlation with spontaneous tail locomotion. We discovered that visual (CS) or tactile stimuli (US) induced $\mathrm{Ca}^{2+}$ transients in a large proportion of the cerebellar neurons $[n=253(22.4 \%)$ and $n=188$ (16.7\%), respectively, of 1127 neurons in which $\mathrm{Ca}^{2+}$ activity was detected] (Fig. 2). Examining the CS and US sensitivity at five focal planes (10-50 $\mu \mathrm{m}$ below the surface) revealed that topological organization of the CS- and US-evoked neurons was heterogeneous: the location of visually driven cells was biased toward the medial
( $p=0.014, \chi^{2}$ test $)$ and anterior cerebellum $\left(p<0.001, \chi^{2}\right.$ test $)$, whereas touch-sensitive neurons were predominantly localized in the ventral and anterior cerebellum ( $p<0.001, \chi^{2}$ test) (Fig. $2 B)$. We found only 24 neurons $(2.12 \%)$ that responded to both the CS and the US.

\section{Learning selectively enhances CS-evoked activity in the cerebellum}

To examine whether learning modulates CS-related activity in the cerebellum, we compared the $\mathrm{Ca}^{2+}$ transients elicited by visual stimulation during the first and seventh trials of conditioning (Fig. 3). Presentation of the CS alone (Fig. 3A) did not significantly alter visual responses in the cerebellum $[n=98$ neurons (4-27 per fish), seven fish, $p=0.49$, paired $t$ test]. In contrast, after seven conditioning trials, when behavioral expression of learning was most evident, we observed a significantly enhanced response to the CS relative to the first trial $[n=165$ neurons (19-53 per fish), five fish, $p<0.001$ ] (Fig. 3B). Above, we described a small population of neurons that responded to both tactile and visual stimuli. We examined whether additional neurons might acquire responsiveness to both sensory modalities as a result of learning. We identified 12 neurons (5.1\%) that expressed US-only-related responses in the first trial but developed significant CS-related responses by the end of conditioning (Fig. 3C) ( $t$ test, $p<0.05$ ). We found no neurons that manifested the opposite transformation. To examine whether the learninginduced CS responsiveness is specific to cerebellar neurons, we performed similar experiments in the optic tectum, the area that receives direct input from the retina and is rich with visually 

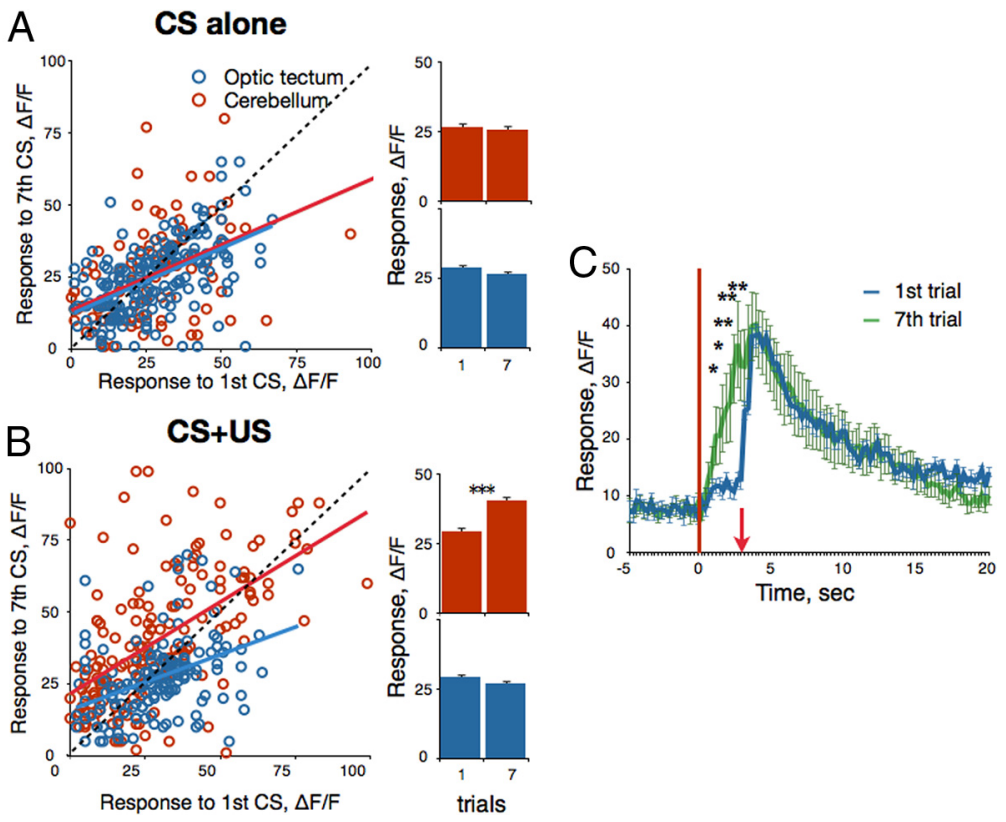

Figure 3. Learning-dependent changes in visually driven activity. $A$, Neurons in the cerebellum (red) and optic tectum (blue) display relatively stable visual responses to a repetitively presented CS. B. Cerebellar, but not tectal, neurons, increase their responses to the $C S$ during conditioning (CS $+U S)$. In $\boldsymbol{A}$ and $\boldsymbol{B}$, each dot represents the response of an individual neuron (peak height) to the (S in trial 7 as a function of the response in trial 1. Color lines are linear trend lines. Bars on the right depict averaged responses in trials 1 and $7 . \Delta F / F_{\text {min }^{\prime}}$ percentage $\pm S E M ;{ }^{* * *} p<0.001$. C, Some neurons responded to the US only at the beginning of the training session but developed an additional peak of activity in response to $C S$ after conditioning. An average response of 12 neurons from six fish $\left(\Delta F / F_{\text {min }^{\prime}}\right.$, percentage $\left.\pm S E M\right)$ in the first (blue) and seventh (green) trials is shown. Onsets of the CS and US are represented by the vertical red line and red arrow, respectively. ${ }^{*} p<0.05 ;{ }^{* *} p<0.01$.
A

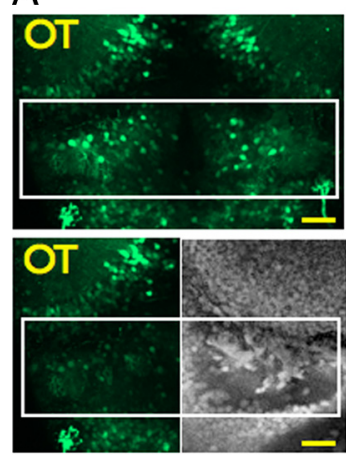

B

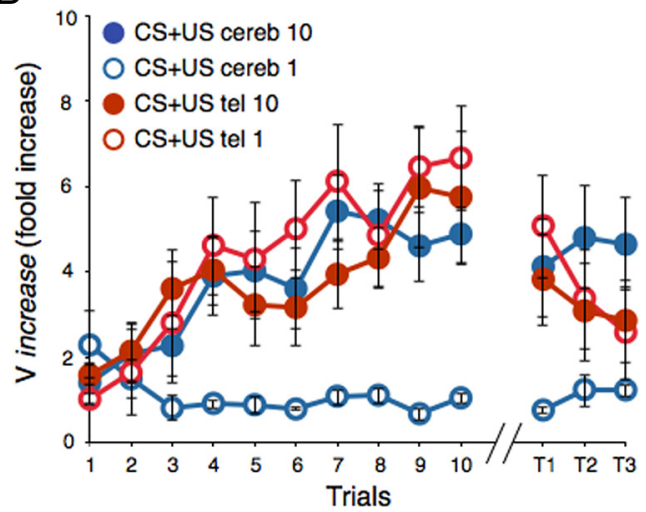

Figure 4. Requirement of the cerebellum, but not the telencephalon, for learning acquisition. $A$, Two-photon ablation of the telencephalon (left) and cerebellum (right). Top is a z-projection of confocal sections taken at depths of $0-40 \mu \mathrm{m}$. Bottom shows the left side of the same fish after ablation and the right side of a fixed brain stained with SYT0 14 (white rectangle shows the ablated region). Scale bar, $20 \mu \mathrm{m} . \boldsymbol{B}$, Graph plots the CR (mean $V_{\text {increase }} \pm \mathrm{SEM}$ ) as a function of trials. Bilateral ablation of the telencephalon performed immediately after the first (open red circles) or 10th (filled red circles) trial did not significantly impair acquisition or retention of memory. Bilateral lesions in the cerebellum after the first trial (open blue circles) blocked acquisition of the CR. Ablation after the 10th trial (filled blue circles) did not affect memory retention but impaired extinction during the three test trials.

evoked activity. Unlike the cerebellum, both CS-alone presentations $[n=184$ neurons ( $17-45$ per fish), seven fish] and conditioning [ $n=151$ neurons (23-39 per fish), five fish] resulted in a modest decrease in mean visual responses in the optic tectum $(p=0.013$ and 0.103 , respectively, paired $t$ test) (Fig. $3 A, B)$.

The CCe is involved in the acquisition, but not the expression, of memory

The observed modulation of cerebellar neuron responses by learning suggested that it is involved in memory acquisition or retention. To examine this possibility, we performed two-photon laser ablation of the CCe after either the first or 10th conditioning trial (Fig. 4A). Ablation of the CCe after the first trial disrupted the acquisition of conditioned responses $(n=6$ fish, $p=0.21$, KW test) (Fig. 4B). Ablation after the 10th trial did not affect memory retention, which was confirmed by the nonsignificant difference between the $\mathrm{CR}$ on the last conditioning trial and test 1 ( $n=10$ fish, $p=0.39$, paired $t$ test). Interestingly, although in intact fish three presentations of the CS alone (T1-T3) led to a rapid extinction of memory (Fig. 1), the larvae that received cerebellar ablations after trial 10 were resistant to extinction. Impaired extinction was confirmed by a repeated-measures ANOVA that revealed no change in the $C R$ of the ablated subjects $(n=10, p=0.91)$ and a two-way ANOVA that revealed a significant interaction between the ablated and intact groups after three extinction trials $(n=$ $10, p=0.046)$. To test whether the observed memory deficit can be specifically attributed to the ablation of cerebellum, we also performed two-photon ablation of the telencephalon after trials 1 or 10 . The acquisition of learning in the fish that underwent ablation after trial $1(n=7)$ or trial $10(n=9)$ was similar to that of the intact (nonablated) group (Fig. $4 \mathrm{~B}$ ). Although memory retention of both the early and late ablation groups was partially diminished ( $p=0.064$, paired $t$ test), it did not differ from that of the intact animals ( $p=0.67, t$ test). As such, it appears that the telencephalon is not required for the acquisition of memory, and its role in memory retention warrants future investigation.

\section{Discussion}

In this study, larval zebrafish learned to associate a moving spot of light (CS) with a touch (US). The acquisition and extinction of conditioned responses occurred rapidly. This effect was specific to paired presentations of the CS and US. Despite the fast learning, the acquired memory did not last longer than $1 \mathrm{~h}$. This relatively short-term memory can be contrasted to long-term memory documented in adult fish of various species (e.g. Eisenberg and Dudai, 2004). It is possible that the short-term nature of the memory reflects a limited capacity of the developing nervous system to establish and maintain long-term memories. Modifications to this or other learning protocols (for example, extensions of the learning phase) may enable the larval fish to maintain memory for longer periods of time. The delicate nature of the preparation, however, precluded us from testing longer conditioning regimes in the present experiments. 
The zebrafish brain has been the subject of many recent comparative physiology studies. Most research has focused on the sensory processing and integrative functions of the optic tectum and olfactory bulb (Friedrich and Laurent, 2001; Niell and Smith, 2005; Sumbre et al., 2008). Other brain areas, such as the cerebellum, have received less attention. We used confocal $\mathrm{Ca}^{2+}$ imaging to optically monitor neural activity in behaving zebrafish larvae. We found two types of cells with different activation properties that are topographically organized: 1) neurons responsive to light located superficially in the CCe; and 2) touch-responsive neurons located more ventrally. This is in agreement with earlier reports of partially segregated visual and somatosensory inputs to CCe in catfish (Lee and Bullock, 1984). If this is the case, then our results raise the question of how sensory information is integrated in the fish cerebellum. It is possible that the integration takes place in a small population of cells that receive both CS and US information.

We discovered that cerebellar neurons display strong facilitation of their responses to the CS after learning. This is consistent with findings in mammals indicating that a large proportion of cerebellar neurons in both cortex and the deep nuclei exhibit conditioning-related increases in firing rate (Gould and Steinmetz, 1996). Although our data demonstrate that conditioning leads to an apparent strengthening of the CS representation in the $\mathrm{CCe}$, it is possible that some neurons undergo inhibition, which is difficult to observe with $\mathrm{Ca}^{2+}$ imaging. Most of the neurons that displayed learning-dependent modifications exhibited facilitation of visual responses. A subset of cells appeared to become bimodal as result of learning. While initially responding only to the US, these neurons exhibited significant CS-related activity during conditioning. This finding suggests that conditioning can drive the integration of the sensory information in a developing brain.

Because of a lack of appropriate transgenic lines, we were unable to identify with certainty the type of cells being monitored. Purkinje cells (PC) are the most likely candidates given their size and abundance at the superficial CCe. However, eurydendroid cells, which constitute the primary cerebellar efferent projections and are intermingled with PCs, cannot be ruled out. Given ample evidence from mammals, it is likely that visually evoked transients in our study are conveyed by mossy fibers, whereas climbing fibers drive tactile responses.

Because the connectivity of the cerebellum in teleost fish is similar to that in mammals (Bae et al., 2009) and some reports have suggested its involvement in learning (Gómez et al., 2010), we hypothesized that the zebrafish cerebellum plays a similar role in our simple conditioning protocol. We found that CCe lesions delivered before conditioning prevented the acquisition of the $\mathrm{CR}$, whereas memory retention and expression remained unaffected when the cerebellum was ablated after conditioning (Fig. 4). Although the role of cerebellum in various conditioning protocols is well established, the brain mechanisms underlying memory extinction are still poorly understood. Recent experimental data implicate both telencephalic structures (the hippocampus) and the cerebellum (nucleus interpositus, in particular) in the extinction of eye-blink conditioning (Robleto and Thompson, 2008). Our data show that lesions of the CCe delivered after learning had reached asymptote significantly in- hibit extinction; in comparison, delayed telencephalic lesions had no effect on extinction. Together, our results indicate that the larval zebrafish CCe is necessary for both the acquisition and the extinction of the CR but is dispensable for retention of memory, which is at variance with the results observed in mammals. Because our lesions spared the valvula and the caudal lobe that have been reported to be important in the modulation of visually guided behavior (Yanagihara et al., 1993), the most plausible explanation for our results is that one (or both) of the spared regions is responsible for retention of the conditioned response. An important area for future studies will be to elucidate the specific cerebellar subregions or cell types involved in different aspects of learning.

\section{References}

Asakawa K, Suster ML, Mizusawa K, Nagayoshi S, Kotani T, Urasaki A, Kishimoto Y, Hibi M, Kawakami K (2008) Genetic dissection of neural circuits by Tol2 transposon-mediated Gal4 gene and enhancer trapping in zebrafish. Proc Natl Acad Sci U S A 105:1255-1260.

Bae YK, Kani S, Shimizu T, Tanabe K, Nojima H, Kimura Y, Higashijima S, Hibi M (2009) Anatomy of zebrafish cerebellum and screen for mutations affecting its development. Dev Biol 330:406-426.

Baier H, Scott EK (2009) Genetic and optical targeting of neural circuits and behavior - zebrafish in the spotlight. Curr Opin Neurobiol 19:553-560.

Burgess HA, Granato M (2007) Sensorimotor gating in larval zebrafish. J Neurosci 27:4984-4994.

Darland T, Dowling JE (2001) Behavioral screening for cocaine sensitivity in mutagenized zebrafish. Proc Natl Acad Sci U S A 98:11691-11696.

Eisenberg M, Dudai Y (2004) Reconsolidation of fresh, remote, and extinguished fear memory in medaka: old fears don't die. Eur J Neurosci 20:3397-3403.

Friedrich RW, Jacobson GA, Zhu P (2010) Circuit neuroscience in zebrafish. Curr Biol 20:R371-R381.

Friedrich RW, Laurent G (2001) Dynamic optimization of odor representations by slow temporal patterning of mitral cell activity. Science 291: 889-894.

Gerlach G, Hodgins-Davis A, Avolio C, Schunter C (2008) Kin recognition in zebrafish: a 24-hour window for olfactory imprinting. Proc Biol Sci 275:2165-2170

Gómez A, Durán E, Salas C, Rodríguez F (2010) Cerebellum lesion impairs eyeblink-like conditioning in goldfish. Neuroscience 166:49-60.

Gould TJ, Steinmetz JE (1996) Changes in rabbit cerebellar cortical and interpositus nucleus activity during acquisition, extinction, and backward classical eyelid conditioning. Neurobiol Learn Mem 65:17-34.

Lee LT, Bullock TH (1984) Sensory representation in the cerebellum of the catfish. Neuroscience 13:157-169.

Niell CM, Smith SJ (2005) Functional imaging reveals rapid development of visual response properties in the zebrafish tectum. Neuron 45:941-951.

Robleto K, Thompson RF (2008) Extinction of a classically conditioned response: Red nucleus and interpositus. J Neurosci 28:2651-2658.

Sison M, Gerlai R (2010) Associative learning in zebrafish (Danio rerio) in the plus maze. Behav Brain Res 207:99-104.

Sumbre G, Muto A, Baier H, Poo MM (2008) Entrained rhythmic activities of neuronal ensembles as perceptual memory of time interval. Nature 456:102-106.

Thompson RF, Steinmetz JE (2009) The role of cerebellum in classical conditioning of discrete behavioral responses. Neuroscience 162:732-755.

Yaksi E, Friedrich RW (2006) Reconstruction of firing rate changes across neuronal populations by temporally deconvolved $\mathrm{Ca}^{2+}$ imaging. Nat Methods 3:377-383.

Yanagihara D, Watanabe S, Takagi S, Mitarai G (1993) Neuroanatomical substrate for the dorsal light response. II. Effects of kainic acid-induced lesions of the valvula cerebelli on the goldfish dorsal light response. Neurosci Res 16:33-37. 